\title{
Prediction of Wheat Stripe Rust Based on Neural Networks
}

\author{
Haiguang Wang ${ }^{*}$ and Zhanhong Ma \\ Department of Plant Pathology, China Agricultural University, Beijing 100193, China \\ wanghaiguang@cau.edu.cn
}

\begin{abstract}
Stripe rust caused by Puccinia striiformis f. sp. tritici, is a devastating wheat disease in the world. The prediction of this disease is very important to make control strategies. In order to figure out suitable prediction methods based on neural networks that could provide accurate prediction information with high stability, the predictions of wheat stripe rust by using backpropagation networks with different transfer functions, training functions and learning functions, radial basis networks, generalized regression networks (GRNNs) and probabilistic neural networks (PNNs) were conducted in this study. The results indicated that suitable backpropagation networks, radial basis networks and GRNNs could be used for the prediction of wheat stripe rust. Good fitting accuracy and prediction accuracy could be obtained by using backpropagation networks with trainlm, trainrp or trainbfg as training function. Radial basis networks had more power than backpropagation networks and GRNNs to predict wheat stripe rust. GRNNs were easier to be used than backpropagation networks. New methods based on neural networks were provided for the prediction of wheat stripe rust.
\end{abstract}

Keywords: Wheat stripe rust, prediction, neural networks, epidemics.

\section{Introduction}

Wheat stripe rust caused by Puccinia striiformis f. sp. tritici, is a very important wheat disease in the world [1]. This disease is widely distributed in eastern and central Asia, western Europe and the pacific coast of North America [1], [2], [3]. The epidemics of this disease could also occur in Oceania, northern and eastern Africa, and South America [1]. In China, in terms of distribution range and destructivity, stripe rust is the most important among the three wheat rusts including leaf rust and stem rust. Stripe rust could occur in all main wheat areas and could cause severe losses in wheat production in China. It is critical to the national food security of China. The epidemics of wheat stripe rust are related to the long distance transports of the pathogen driven by winds. Accurate prediction of this disease is the key to make strategies for effective control.

The prediction of plant disease is to estimate the prevalence of plant disease after a certain time using expert experience or system simulation methods based on epidemic

${ }^{*}$ Corresponding author. 
law of the disease. There have been many reports about the prediction of wheat stripe rust. The methods that were usually used included regression analysis [4], [5], [6], [7], discrimination analysis [8], [9], [10], Markov forecast method [11], principal component analysis [12], grey model forecast method [13], [14] and neural networks [15], [16], [17]. Wheat stripe rust is influenced by various factors, including the amount of pathogen, variety resistance and meteorological conditions. The relationship between wheat stripe rust and these factors is usually nonlinear. Neural networks have good advantages of solving the nonlinear problems. They are composed of interconnecting artificial neurons. They have good adaptive learning ability and nonlinear mapping ability. Neural networks have been successfully applied in various fields, including pattern recognition, image analysis and control systems. Although neural networks have been used for the prediction of wheat stripe rust [15], [16], [17], individual backpropagation network or individual function combination was be used in most cases. To the authors' knowledge, there is not any report about the application of radial basis networks to the prediction of wheat stripe rust. Furthermore, the comparison between prediction performances of different neural networks and the comparison between prediction performances of backpropagation networks combined with different transfer functions, training functions and learning functions have not been reported yet.

In order to select prediction methods based on neural networks with good prediction performance and prediction accuracy and provide disease information for making control strategies and taking control measures, the predictions of wheat stripe rust based on backpropagation networks with different transfer functions, training functions and learning functions, radial basis networks, and two variants of radial basis networks, generalized regression networks (GRNNs) and probabilistic neural networks (PNNs), were conducted and their prediction effects were compared in this study.

\section{Materials and Methods}

\subsection{Materials}

The data used for analysis in this study were colleted from [18]. They consisted of the data of disease prevalence of wheat stripe rust and related meteorological data in Hanzhong in Shannxi Province during the period from 1974 to 1997. In detail, they included the amount of stripe rust pathogen in autumn, the amount of stripe rust pathogen in spring, average temperature in January, average temperature in February, average temperature in March, average temperature in April, average temperature in November, average temperature in December, precipitation in January, precipitation in February, precipitation in March, precipitation in April, precipitation in November, precipitation in December, the proportion of susceptible variety area and disease prevalence in adult stage of wheat. Among these factors, the amount of stripe rust pathogen in autumn and related data in November and December referred to the data in the preceding year. The fifteen influencing factors were normalized. The data set contained the fifteen normalized factors and disease prevalence of wheat stripe rust 
was regarded as Data set 1 . In the data set, disease prevalence of wheat stripe rust was divided into five classes represented by $1,2,3,4$ and 5, respectively. The data set was regarded as Data set 2 while Class 1, 2, 3, 4 and 5 were expressed as $\left(\begin{array}{lllll}0 & 0 & 0 & 0 & 1\end{array}\right),\left(\begin{array}{lll}0 & 0\end{array}\right.$ $\left.\begin{array}{lll}0 & 1 & 0\end{array}\right),\left(\begin{array}{lllll}0 & 0 & 1 & 0 & 0\end{array}\right),\left(\begin{array}{lllll}0 & 1 & 0 & 0 & 0\end{array}\right)$ and $\left(\begin{array}{lllll}1 & 0 & 0 & 0 & 0\end{array}\right)$, respectively.

In the literature [18], the amount of stripe rust pathogen in spring, the amount of stripe rust pathogen in autumn, the proportion of susceptible variety area, precipitation in April and average temperature in April were selected as the main factors influencing disease prevalence from the fifteen factors by using stepwise regression analysis. The five selected factors were normalized. The data set contained the five normalized factors and disease prevalence of wheat stripe rust represented by $1,2,3,4$ or 5, was regarded as Data set 3. The data set was regarded as Data set 4

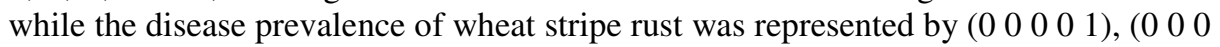
$10),\left(\begin{array}{lllll}0 & 0 & 1 & 0 & 0\end{array}\right),\left(\begin{array}{lllll}0 & 1 & 0 & 0 & 0\end{array}\right)$ or $\left(\begin{array}{lllll}1 & 0 & 0 & 0 & 0\end{array}\right)$.

\subsection{Methods}

Under MATLAB environment, backpropagation networks were used to process Data set 2 and Data set 4, radial basis networks and GRNNs were used to process Data set 1, Data set 2, Data set 3 and Data set 4, and PNNs were used to process Data set 1 and Data set 3. During data processing, disease prevalences were used as outputs and the other variables were used as inputs. For the data sets, the data in 1974-1993 were considered as the training sets and the data in 1994-1997 were considered as the testing sets. The prevalences of wheat stripe rust in 1974-1993 in Hanzhong in Shannxi Province were fitted and that in 1994-1997 were predicted. Firstly, the training sets were trained to acquire optimized neural networks. And then the neural networks were used to predict output values of the testing sets, and meanwhile the training sets were also fitted. The fitting and prediction results obtained were compared with actual disease prevalences and then fitting accuracy and prediction accuracy were obtained, respectively.

One-hidden-layer backpropagation networks were constructed. Three common transfer functions including logsig, tansig and purelin were used in the networks. Since the outputs of the networks were between 0 and 1 , the output layer used the logsigmoid transfer function (logsig). The networks used trainlm, traingd, traingdm, trainrp and trainbfg for training. Learngd and learngdm were used as learning functions. Different combinations of transfer functions, training functions and learning functions were recorded as BP1, BP2, BP3, .., BP30, and were shown as in Table 1. For Data set 2, fifteen neurons were used in the input layer and five neurons were used in the output layer. For Data set 4, five neurons were used in the input layer and five neurons were used in the output layer. Maximum number of epochs to train for the backpropagation networks was 5000. The neuron number in the hidden layer was assumed to be 1 to 20 with step size 1 .

Radial basis networks, GRNNs and PNNs were used to fit the training sets and predict the disease prevalences using the testing sets. Spreads of radial basis functions for radial basis networks, GRNNs and PNNs were assumed to be 0.1 to 2.0 with step size 0.1 . 
Table 1. Different combinations of transfer functions, training functions and learning functions for backpropagation networks

\begin{tabular}{ccccc}
\hline & \multicolumn{1}{c}{ Transfer function } & Training function & Learning function \\
Tag & Hidden layer & Output layer & & \\
\hline BP1 & tansig & logsig & trainlm & learngdm \\
BP2 & purelin & logsig & trainlm & learngdm \\
BP3 & logsig & logsig & trainlm & learngdm \\
BP4 & tansig & logsig & trainlm & learngd \\
BP5 & purelin & logsig & trainlm & learngd \\
BP6 & logsig & logsig & trainlm & learngd \\
BP7 & tansig & logsig & traingd & learngdm \\
BP8 & purelin & logsig & traingd & learngdm \\
BP9 & logsig & logsig & traingd & learngdm \\
BP10 & tansig & logsig & traingd & learngd \\
BP11 & purelin & logsig & traingd & learngd \\
BP12 & logsig & logsig & traingd & learngd \\
BP13 & tansig & logsig & traingdm & learngdm \\
BP14 & purelin & logsig & traingdm & learngdm \\
BP15 & logsig & logsig & traingdm & learngdm \\
BP16 & tansig & logsig & traingdm & learngd \\
BP17 & purelin & logsig & traingdm & learngd \\
BP18 & logsig & logsig & traingdm & learngd \\
BP19 & tansig & logsig & trainrp & learngdm \\
BP20 & purelin & logsig & trainrp & learngdm \\
BP21 & logsig & logsig & trainrp & learngdm \\
BP22 & tansig & logsig & trainrp & learngd \\
BP23 & purelin & logsig & trainrp & learngd \\
BP24 & logsig & logsig & trainrp & learngd \\
BP25 & tansig & logsig & trainbfg & learngdm \\
BP26 & purelin & logsig & trainbfg & learngdm \\
BP27 & logsig & logsig & trainbfg & learngdm \\
BP28 & tansig & logsig & trainbfg & learngd \\
BP29 & purelin & logsig & trainbfg & learngd \\
BP30 & logsig & logsig & trainbfg & learngd \\
\hline & & & & \\
\hline
\end{tabular}

\section{$3 \quad$ Results and Analysis}

The data sets were processed using the methods as shown above. The results of the neural networks with fitting accuracy $\geq 75 \%$ and prediction accuracy $\geq 50 \%$ were picked out and were shown in Table 2, Table 3, Table 4, Table 5, Table 6 and Table 7 , respectively. 
Using the backpropagation networks, for either Data set 2 or Data set 4 , the selected combinations with good fitting accuracy and prediction accuracy were BP1, BP2, BP3, BP4, BP5, BP6, BP19, BP20, BP21, BP22, BP23, BP24, BP25, BP28, $\mathrm{BP} 29$ and BP30. Among these combinations, the training functions of BP1, BP2, BP3, BP4, BP5 and BP6 were trainlm, that of BP19, BP20, BP21, BP22, BP23, BP24 and BP25 were trainrp, and that of BP28, BP29 and BP30 were trainbfg. When the training function was traingd or traingdm, good fitting accuracy and prediction accuracy could not be obtained using the backpropagation networks. So it is important to choose suitable training function for backpropagation networks.

As Table 2 shown, for Data set 2, the fitting accuracies were all $100 \%$ and the prediction accuracies were all $75 \%$ while using BP1 with the number of neurons in the hidden layer equal to 3 or 11, BP3 with the number of neurons in the hidden layer equal to 10,12 or 17, BP6 with the number of neurons in the hidden layer equal to 16 , BP19 with the number of neurons in the hidden layer equal to 4, BP21 with the number of neurons in the hidden layer equal to 5, BP24 with the number of neurons in the hidden layer equal to 10 or 19 , and BP28 with the number of neurons in the hidden layer equal to 5 .

For Data set 4 , the fitting results and the prediction results of the backpropagation networks were shown as in Table 3. Fitting accuracies were all $100 \%$ and prediction accuracies were all $75 \%$ while using BP4 with the number of neurons in the hidden layer equal to 9 and BP22 with the number of neurons in the hidden layer equal to 8 besides the combinations as the same as those with the same number of neurons in the hidden layer for Data set 2.

The results indicated that backpropagation networks could be used for the prediction of wheat stripe rust. When using this method, the inputs could be the data of all the factors obtained in the investigations or be the data of selected main factors. Otherwise, it would take a longer time to build suitable networks when using the data of all the influencing factors obtained.

The fitting results and the prediction results of radial basis networks were shown as in Table 4 and Table 5. The fitting accuracies were all $100 \%$ for the selected networks. The results implied that radial basis networks have good adaptive learning ability and stable prediction ability. For Data set 1, the prediction accuracy was $75 \%$ when the value of spread was $0.8,0.9,1.0$ or 1.1 . For Data set 2 , the prediction accuracies were all 50\% while using the selected networks. For Data set 3, the lowest prediction accuracy was $50 \%$ and the highest prediction accuracy was $100 \%$. The prediction accuracy was $75 \%$ when the value of spread was $0.3,0.4,0.5,1.5,1.6,1.7$, $1.8,1.9$ or 2.0 . The prediction accuracy was $100 \%$ when the value of spread was 0.6 to 1.4 . For Data set 4 , the prediction accuracy was $75 \%$ when the value of spread was $0.3,1.2,1.3,1.4$ or 1.5 . The results indicated that radial basis networks have high prediction ability for the disease. In practical applications, it is better to choose the key influencing factors as the inputs of the networks to improve the run speed of the programs, prediction accuracy, and performance stability. 
Table 2. The fitting results and the prediction results of the backpropagation networks for Data set 2

\begin{tabular}{|c|c|c|c|c|c|c|c|}
\hline Tag & $\begin{array}{c}\text { Number of neurons in the } \\
\text { hidden layer }\end{array}$ & $\begin{array}{c}\text { Fitting } \\
\text { accuracy }\end{array}$ & $\begin{array}{c}\text { Prediction } \\
\text { accuracy }\end{array}$ & Tag & $\begin{array}{c}\text { Number of neurons in } \\
\text { the hidden layer }\end{array}$ & $\begin{array}{c}\text { Fitting } \\
\text { accuracy }\end{array}$ & $\begin{array}{c}\text { Prediction } \\
\text { accuracy }\end{array}$ \\
\hline BP1 & 1 & $80 \%$ & $50 \%$ & BP19 & 4 & $100 \%$ & $75 \%$ \\
\hline BP1 & 4,5 & $85 \%$ & $50 \%$ & |BP20 & $\begin{array}{c}10,11,13,15,16 \\
17,18\end{array}$ & $100 \%$ & $50 \%$ \\
\hline BP1 & 6 & $95 \%$ & $50 \%$ & |BP21 & $\begin{array}{c}2,4,14,15,16,17 \\
19,20\end{array}$ & $95 \%$ & $50 \%$ \\
\hline BP1 & $\begin{array}{c}8,9,10,12,13,14,15 \\
16,17,18,19,20\end{array}$ & $100 \%$ & $50 \%$ & |BP21 & $\begin{array}{c}3,6,7,8,9,10,11 \\
12,13,18\end{array}$ & $100 \%$ & $50 \%$ \\
\hline BP1 & 7 & $95 \%$ & $75 \%$ & BP21 & 5 & $100 \%$ & $75 \%$ \\
\hline BP1 & 3,11 & $100 \%$ & $75 \%$ & |BP22 & $2,4,5,10,12,17,18$ & $95 \%$ & $50 \%$ \\
\hline $\mathrm{BP} 2$ & $1,2,4,13$ & $85 \%$ & $50 \%$ & |BP22 & $\begin{array}{c}6,7,9,11,13,14 \\
15,16,19,20\end{array}$ & $100 \%$ & $50 \%$ \\
\hline BP2 & 3 & $95 \%$ & $50 \%$ & | BP22 & 8 & $100 \%$ & $75 \%$ \\
\hline BP2 & 6 & $100 \%$ & $50 \%$ & BP23 & 10 & $90 \%$ & $50 \%$ \\
\hline $\mathrm{BP} 3$ & 1,7 & $85 \%$ & $50 \%$ & BP23 & $\begin{array}{c}5,7,12,13,14,15 \\
17\end{array}$ & $95 \%$ & $50 \%$ \\
\hline BP3 & 9 & $90 \%$ & $50 \%$ & BP23 & $\begin{array}{c}4,6,8,9,11,16 \\
18,19,20\end{array}$ & $100 \%$ & $50 \%$ \\
\hline BP3 & $3,4,11,13,16$ & $95 \%$ & $50 \%$ & BP24 & 1 & $85 \%$ & $50 \%$ \\
\hline BP3 & $\begin{array}{c}2,5,8,14,15,18,19, \\
20\end{array}$ & $100 \%$ & $50 \%$ & |BP24 & $\begin{array}{c}2,3,4,9,15,16 \\
20\end{array}$ & $95 \%$ & $50 \%$ \\
\hline $\mathrm{BP} 3$ & $10,12,17$ & $100 \%$ & $75 \%$ & |BP24 & $\begin{array}{c}6,8,11,12,13,14 \\
17,18\end{array}$ & $100 \%$ & $50 \%$ \\
\hline BP4 & 7 & $75 \%$ & $50 \%$ & BP24 & 10,19 & $100 \%$ & $75 \%$ \\
\hline BP4 & $3,4,5,17$ & $85 \%$ & $50 \%$ & BP25 & 15 & $80 \%$ & $75 \%$ \\
\hline $\mathrm{BP} 4$ & $\begin{array}{c}2,6,8,9,10,11,16 \\
18,19,20\end{array}$ & $100 \%$ & $50 \%$ & |BP25 & 7 & $85 \%$ & $50 \%$ \\
\hline BP5 & 1 & $75 \%$ & $50 \%$ & BP25 & 4,18 & $95 \%$ & $50 \%$ \\
\hline BP5 & 2,8 & $80 \%$ & $50 \%$ & |BP25 & $9,11,14,17,20$ & $100 \%$ & $50 \%$ \\
\hline BP5 & $3,5,9$ & $85 \%$ & $50 \%$ & |BP28 & $7,11,19$ & $85 \%$ & $50 \%$ \\
\hline BP5 & 4,20 & $90 \%$ & $50 \%$ & BP28 & 1 & $90 \%$ & $75 \%$ \\
\hline BP5 & $6,13,15$ & $95 \%$ & $50 \%$ & |BP28 & 4,15 & $95 \%$ & $50 \%$ \\
\hline BP5 & $7,10,11,17,19$ & $100 \%$ & $50 \%$ & BP28 & $12,13,14,17$ & $100 \%$ & $50 \%$ \\
\hline BP6 & 11,19 & $90 \%$ & $50 \%$ & BP28 & 5 & $100 \%$ & $75 \%$ \\
\hline BP6 & 13 & $90 \%$ & $75 \%$ & |BP29 & 5 & $85 \%$ & $50 \%$ \\
\hline BP6 & 4 & $95 \%$ & $50 \%$ & BP29 & 10 & $100 \%$ & $50 \%$ \\
\hline BP6 & $\begin{array}{c}3,5,6,7,9,10,12,14 \\
15,17,18\end{array}$ & $100 \%$ & $50 \%$ & BP30 & 20 & $85 \%$ & $50 \%$ \\
\hline BP6 & 16 & $100 \%$ & $75 \%$ & BP30 & $7,13,16$ & $90 \%$ & $50 \%$ \\
\hline BP19 & 2 & $90 \%$ & $50 \%$ & BP30 & 8 & $95 \%$ & $50 \%$ \\
\hline BP19 & $3,8,13$ & $95 \%$ & $50 \%$ & BP30 & 3 & $100 \%$ & $50 \%$ \\
\hline BP19 & $\begin{array}{c}5,6,7,9,10,11,12, \\
14,15,16,17,20\end{array}$ & $100 \%$ & $50 \%$ & & & & \\
\hline
\end{tabular}


Table 3. The fitting results and the prediction results of the backpropagation networks for Data set 4

\begin{tabular}{|c|c|c|c|c|c|c|c|}
\hline Tag & $\begin{array}{l}\text { Number of neurons in } \\
\text { the hidden layer }\end{array}$ & $\begin{array}{c}\text { Fitting } \\
\text { accuracy }\end{array}$ & $\begin{array}{c}\text { Prediction } \\
\text { accuracy }\end{array}$ & Tag & $\begin{array}{l}\text { Number of neurons in } \\
\text { the hidden layer }\end{array}$ & $\begin{array}{c}\text { Fitting } \\
\text { accuracy }\end{array}$ & $\begin{array}{r}\text { Prediction } \\
\text { accuracy } \\
\end{array}$ \\
\hline BP1 & 1 & $80 \%$ & $50 \%$ & BP19 & 4 & $100 \%$ & $75 \%$ \\
\hline BP1 & 4,5 & $85 \%$ & $50 \%$ & BP19 & $\begin{array}{c}5,6,7,9,10,11,12 \\
14,15,16,17,20\end{array}$ & $100 \%$ & $50 \%$ \\
\hline BP1 & 6 & $95 \%$ & $50 \%$ & BP20 & $\begin{array}{c}10,11,13,15,16 \\
17,18\end{array}$ & $100 \%$ & $50 \%$ \\
\hline BP1 & 7 & $95 \%$ & $75 \%$ & BP21 & $\begin{array}{c}2,4,14,15,16,17 \\
19,20\end{array}$ & $95 \%$ & $50 \%$ \\
\hline $\mathrm{BP} 1$ & $\begin{array}{c}8,9,10,12,13,14,15 \\
16,17,18,19,20\end{array}$ & $100 \%$ & $50 \%$ & BP21 & $\begin{array}{c}3,6,7,8,9,10,11 \\
12,13,18\end{array}$ & $100 \%$ & $50 \%$ \\
\hline $\mathrm{BP} 1$ & 3,11 & $100 \%$ & $75 \%$ & BP21 & 5 & $100 \%$ & $75 \%$ \\
\hline $\mathrm{BP} 2$ & $1,2,4,13$ & $85 \%$ & $50 \%$ & BP22 & $\begin{array}{c}2,4,5,10,12,17, \\
18\end{array}$ & $95 \%$ & $50 \%$ \\
\hline BP2 & 3 & $95 \%$ & $50 \%$ & BP22 & $\begin{array}{c}6,7,9,11,13,14 \\
15,16,19,20\end{array}$ & $100 \%$ & $50 \%$ \\
\hline BP2 & 6 & $100 \%$ & $50 \%$ & BP22 & 8 & $100 \%$ & $75 \%$ \\
\hline $\mathrm{BP} 3$ & 1,7 & $85 \%$ & $50 \%$ & BP23 & 10 & $80 \%$ & $50 \%$ \\
\hline BP3 & $\begin{array}{c}2,5,8,14,15,18,19 \\
20\end{array}$ & $100 \%$ & $50 \%$ & BP23 & $\begin{array}{c}5,7,12,13,14,15 \\
17\end{array}$ & $95 \%$ & $50 \%$ \\
\hline BP3 & 9 & $90 \%$ & $50 \%$ & BP23 & $\begin{array}{c}4,6,8,9,11,16,18 \\
19,20\end{array}$ & $100 \%$ & $50 \%$ \\
\hline $\mathrm{BP} 3$ & $3,4,11,13,16$ & $95 \%$ & $50 \%$ & BP24 & 1 & $85 \%$ & $50 \%$ \\
\hline BP3 & $10,12,17$ & $100 \%$ & $75 \%$ & BP24 & $2,3,4,9,15,16,20$ & $95 \%$ & $50 \%$ \\
\hline BP4 & $\begin{array}{c}2,6,8,10,11,16,18 \\
19,20\end{array}$ & $100 \%$ & $50 \%$ & BP24 & $\begin{array}{c}6,8,11,12,13,14 \\
17,18\end{array}$ & $100 \%$ & $50 \%$ \\
\hline BP4 & 7 & $75 \%$ & $50 \%$ & BP24 & 10,19 & $100 \%$ & $75 \%$ \\
\hline BP4 & $3,4,5,17$ & $85 \%$ & $50 \%$ & BP25 & 15 & $80 \%$ & $75 \%$ \\
\hline BP4 & 9 & $100 \%$ & $75 \%$ & BP25 & 7 & $85 \%$ & $50 \%$ \\
\hline BP5 & 1 & $75 \%$ & $50 \%$ & BP25 & 4,18 & $95 \%$ & $50 \%$ \\
\hline BP5 & 2,8 & $80 \%$ & $50 \%$ & BP25 & $9,11,14,17,20$ & $100 \%$ & $50 \%$ \\
\hline BP5 & $3,5,9$ & $85 \%$ & $50 \%$ & BP28 & $7,11,19$ & $85 \%$ & $50 \%$ \\
\hline BP5 & 4,20 & $90 \%$ & $50 \%$ & BP28 & 1 & $90 \%$ & $75 \%$ \\
\hline BP5 & $6,13,15$ & $95 \%$ & $50 \%$ & BP28 & 4,15 & $95 \%$ & $50 \%$ \\
\hline BP5 & $7,10,11,17,19$ & $100 \%$ & $50 \%$ & BP28 & $12,13,14,17$ & $100 \%$ & $50 \%$ \\
\hline BP6 & 11,19 & $90 \%$ & $50 \%$ & BP28 & 5 & $100 \%$ & $75 \%$ \\
\hline BP6 & 13 & $90 \%$ & $75 \%$ & BP29 & 5 & $85 \%$ & $50 \%$ \\
\hline BP6 & 4 & $95 \%$ & $50 \%$ & BP29 & 10 & $100 \%$ & $50 \%$ \\
\hline BP6 & $\begin{array}{c}3,5,6,7,8,9,10,12 \\
14,15,17,18\end{array}$ & $100 \%$ & $50 \%$ & BP30 & 20 & $85 \%$ & $50 \%$ \\
\hline BP6 & 16 & $100 \%$ & $75 \%$ & BP30 & $7,13,16$ & $90 \%$ & $50 \%$ \\
\hline BP19 & 2 & $90 \%$ & $50 \%$ & BP30 & 8 & $95 \%$ & $50 \%$ \\
\hline BP19 & $3,8,13$ & $95 \%$ & $50 \%$ & BP30 & 3 & $100 \%$ & $50 \%$ \\
\hline
\end{tabular}


The fitting results and the prediction results of GRNNs were shown as in Table 6 . For Data set 1 including the fifteen influencing factors, the fitting accuracy was 100\% and the prediction accuracy was $75 \%$ while the value of spread was equal to 0.3 . For Data set 3 including the five selected factors, the fitting accuracy was $100 \%$ and the prediction accuracy was $75 \%$ while the value of spread was equal to 0.1 or 0.2 . And for Data set 3, there was one exception that the fitting accuracy was only $80 \%$, but the prediction accuracy was $75 \%$ while the value of spread was equal to 0.4 . For Data set 2 including the fifteen influencing factors, the fitting accuracy was $100 \%$ and the prediction accuracy was $50 \%$ while the value of spread was equal to $0.2,0.3$ or 0.4 . For Data set 4 including the five selected factors, the fitting accuracy was $100 \%$ and the prediction accuracy was $50 \%$ only when the value of spread was 0.1 . The results showed that GRNNs could be used as a method to predict wheat stripe rust and that the prediction ability when disease prevalence of wheat stripe rust was expressed as 1 , $2,3,4$ or 5 was higher than that when disease prevalence was expressed as $\left(\begin{array}{lllll}0 & 0 & 0 & 0 & 1\end{array}\right)$, $\left(\begin{array}{lllll}0 & 0 & 0 & 1 & 0\end{array}\right),\left(\begin{array}{lllll}0 & 0 & 1 & 0 & 0\end{array}\right),\left(\begin{array}{lllll}0 & 1 & 0 & 0 & 0\end{array}\right)$ or $\left(\begin{array}{lllll}1 & 0 & 0 & 0 & 0\end{array}\right)$. Therefore, disease prevalence could be expressed as 1, 2, 3, 4 or 5 when the prediction of wheat stripe rust is conducted using GRNNs.

Table 4. The fitting results and the prediction results of radial basis networks for Data set 1 and Data set 2

\begin{tabular}{cccc}
\hline Data set & Spread & Fitting accuracy & Prediction accuracy \\
\hline Data set 1 & 0.1 & $100 \%$ & $50 \%$ \\
Data set 1 & 0.2 & $100 \%$ & $50 \%$ \\
Data set 1 & 0.3 & $100 \%$ & $50 \%$ \\
Data set 1 & 0.4 & $100 \%$ & $50 \%$ \\
Data set 1 & 0.6 & $100 \%$ & $50 \%$ \\
Data set 1 & 0.8 & $100 \%$ & $75 \%$ \\
Data set 1 & 0.9 & $100 \%$ & $75 \%$ \\
Data set 1 & 1.0 & $100 \%$ & $75 \%$ \\
Data set 1 & 1.1 & $100 \%$ & $75 \%$ \\
Data set 1 & 1.2 & $100 \%$ & $50 \%$ \\
Data set 1 & 1.3 & $100 \%$ & $50 \%$ \\
Data set 1 & 1.4 & $100 \%$ & $50 \%$ \\
Data set 1 & 1.5 & $100 \%$ & $50 \%$ \\
Data set 1 & 1.6 & $100 \%$ & $50 \%$ \\
Data set 1 & 1.7 & $100 \%$ & $50 \%$ \\
Data set 1 & 1.8 & $100 \%$ & $50 \%$ \\
Data set 1 & 1.9 & $100 \%$ & $50 \%$ \\
Data set 1 & 2.0 & $100 \%$ & $50 \%$ \\
Data set 2 & 0.1 & $100 \%$ & $50 \%$ \\
Data set 2 & 0.2 & $100 \%$ & $50 \%$ \\
Data set 2 & 0.3 & $100 \%$ & $50 \%$ \\
Data set 2 & 0.4 & $100 \%$ & $50 \%$ \\
Data set 2 & 0.6 & $100 \%$ & $50 \%$ \\
Data set 2 & 1.9 & $100 \%$ & $50 \%$ \\
Data set 2 & 2.0 & $100 \%$ & $50 \%$ \\
\hline
\end{tabular}


When PNNs were used to process the data of wheat stripe rust, the highest fitting accuracy was $100 \%$ and the highest prediction accuracy was 50\% (as shown in Table 7). PNNs showed relatively low prediction ability. For Data set 1 , the fitting accuracy was $100 \%$ and the prediction accuracy was $50 \%$ when the value of spread was 0.1 , $0.2,0.3$ or 0.4 . For Data set 3 , the fitting accuracy was $100 \%$ and the prediction accuracy was $50 \%$ when the value of spread was 0.1 . The fitting accuracy had a tendency to decrease with the value of spread increasing.

Table 5. The fitting results and the prediction results of radial basis networks for Data set 3 and Data set 4

\begin{tabular}{cccc||cccc}
\hline Data set & Spread & $\begin{array}{c}\text { Fitting } \\
\text { accuracy }\end{array}$ & $\begin{array}{c}\text { Prediction } \\
\text { accuracy }\end{array}$ & Data set & Spread & $\begin{array}{c}\text { Fitting } \\
\text { accuracy }\end{array}$ & $\begin{array}{c}\text { Prediction } \\
\text { accuracy }\end{array}$ \\
\hline Data set 3 & 0.1 & $100 \%$ & $50 \%$ & Data set 4 & 0.1 & $100 \%$ & $50 \%$ \\
Data set 3 & 0.2 & $100 \%$ & $50 \%$ & Data set 4 & 0.2 & $100 \%$ & $50 \%$ \\
Data set 3 & 0.3 & $100 \%$ & $75 \%$ & Data set 4 & 0.3 & $100 \%$ & $75 \%$ \\
Data set 3 & 0.4 & $100 \%$ & $75 \%$ & Data set 4 & 0.4 & $100 \%$ & $50 \%$ \\
Data set 3 & 0.5 & $100 \%$ & $75 \%$ & Data set 4 & 0.5 & $100 \%$ & $50 \%$ \\
Data set 3 & 0.6 & $100 \%$ & $100 \%$ & Data set 4 & 0.6 & $100 \%$ & $50 \%$ \\
Data set 3 & 0.7 & $100 \%$ & $100 \%$ & Data set 4 & 0.7 & $100 \%$ & $50 \%$ \\
Data set 3 & 0.8 & $100 \%$ & $100 \%$ & Data set 4 & 0.8 & $100 \%$ & $50 \%$ \\
Data set 3 & 0.9 & $100 \%$ & $100 \%$ & Data set 4 & 0.9 & $100 \%$ & $50 \%$ \\
Data set 3 & 1.0 & $100 \%$ & $100 \%$ & Data set 4 & 1.0 & $100 \%$ & $50 \%$ \\
Data set 3 & 1.1 & $100 \%$ & $100 \%$ & Data set 4 & 1.1 & $100 \%$ & $50 \%$ \\
Data set 3 & 1.2 & $100 \%$ & $100 \%$ & Data set 4 & 1.2 & $100 \%$ & $75 \%$ \\
Data set 3 & 1.3 & $100 \%$ & $100 \%$ & Data set 4 & 1.3 & $100 \%$ & $75 \%$ \\
Data set 3 & 1.4 & $100 \%$ & $100 \%$ & Data set 4 & 1.4 & $100 \%$ & $75 \%$ \\
Data set 3 & 1.5 & $100 \%$ & $75 \%$ & Data set 4 & 1.5 & $100 \%$ & $75 \%$ \\
Data set 3 & 1.6 & $100 \%$ & $75 \%$ & Data set 4 & 1.6 & $100 \%$ & $50 \%$ \\
Data set 3 & 1.7 & $100 \%$ & $75 \%$ & Data set 4 & 1.7 & $100 \%$ & $50 \%$ \\
Data set 3 & 1.8 & $100 \%$ & $75 \%$ & Data set 4 & 1.8 & $100 \%$ & $50 \%$ \\
Data set 3 & 1.9 & $100 \%$ & $75 \%$ & Data set 4 & 1.9 & $100 \%$ & $50 \%$ \\
Data set 3 & 2.0 & $100 \%$ & $75 \%$ & Data set 4 & 2.0 & $100 \%$ & $50 \%$ \\
\hline
\end{tabular}

Table 6. The fitting results and the prediction results of GRNNs

\begin{tabular}{cccc}
\hline Data set & Spread & Fitting accuracy & Prediction accuracy \\
\hline Data set 1 & 0.2 & $100 \%$ & $50 \%$ \\
Data set 1 & 0.3 & $100 \%$ & $75 \%$ \\
Data set 1 & 0.4 & $100 \%$ & $50 \%$ \\
Data set 1 & 0.5 & $95 \%$ & $75 \%$ \\
Data set 1 & 0.6 & $95 \%$ & $50 \%$ \\
Data set 2 & 0.2 & $100 \%$ & $50 \%$ \\
Data set 2 & 0.3 & $100 \%$ & $50 \%$ \\
Data set 2 & 0.4 & $100 \%$ & $50 \%$
\end{tabular}


Table 6. (Continued)

$\begin{array}{lccc}\text { Data set 2 } & 0.5 & 95 \% & 50 \% \\ \text { Data set 2 } & 0.6 & 95 \% & 50 \% \\ \text { Data set 2 } & 0.7 & 85 \% & 50 \% \\ \text { Data set 2 } & 0.8 & 85 \% & 50 \% \\ \text { Data set 3 } & 0.1 & 100 \% & 75 \% \\ \text { Data set 3 } & 0.2 & 100 \% & 75 \% \\ \text { Data set 3 } & 0.3 & 90 \% & 75 \% \\ \text { Data set 3 } & 0.4 & 80 \% & 100 \% \\ \text { Data set 4 } & 0.1 & 100 \% & 50 \% \\ \text { Data set 4 } & 0.2 & 95 \% & 50 \% \\ \text { Data set 4 } & 0.3 & 95 \% & 50 \% \\ \text { Data set 4 } & 0.4 & 80 \% & 50 \% \\ \text { Data set 4 } & 0.5 & 80 \% & 50 \%\end{array}$

Table 7. The fitting results and the prediction results of PNNs for Data set 1 and Data set 3

\begin{tabular}{cccc}
\hline Data set & Spread & Fitting accuracy & Prediction accuracy \\
\hline Data set 1 & 0.1 & $100 \%$ & $50 \%$ \\
Data set 1 & 0.2 & $100 \%$ & $50 \%$ \\
Data set 1 & 0.3 & $100 \%$ & $50 \%$ \\
Data set 1 & 0.4 & $100 \%$ & $50 \%$ \\
Data set 1 & 0.5 & $95 \%$ & $50 \%$ \\
Data set 1 & 0.6 & $95 \%$ & $50 \%$ \\
Data set 1 & 0.7 & $85 \%$ & $50 \%$ \\
Data set 1 & 0.8 & $80 \%$ & $50 \%$ \\
Data set 3 & 0.1 & $100 \%$ & $50 \%$ \\
Data set 3 & 0.2 & $95 \%$ & $50 \%$ \\
Data set 3 & 0.3 & $95 \%$ & $50 \%$ \\
Data set 3 & 0.4 & $80 \%$ & $50 \%$ \\
Data set 3 & 0.5 & $80 \%$ & $50 \%$ \\
\hline
\end{tabular}

\section{Conclusion and Discussion}

In this study, backpropagation networks with different transfer functions, training functions and learning functions, radial basis networks, GRNNs and PNNs were applied to predict wheat stripe rust, and comparison of prediction effects of these methods were also conducted. The results showed that good fitting accuracy and prediction accuracy could be achieved using suitable backpropagation networks, radial basis networks and GRNNs, and that the methods based on these three algorithms of neural networks could be used for the prediction of wheat stripe rust. In contrast, radial basis networks had more power to predict wheat stripe rust than backpropagation networks and GRNNs, and the programs of GRNNs were simpler than backpropagation networks and needed less time to run. The backpropagation 
networks using traingd or traingdm as the training function could not fit the data of wheat stripe rust well and could not make accurate predictions. The studies on using neural networks to predict wheat stripe rust reported before mainly used backpropagation networks [15], [16], [17]. This study provides new approaches based neural networks for the prediction of this disease.

Many studies on the prediction of wheat stripe rust have been conducted and many methods have been applied for the prediction of this disease. However, some kinds of problems such as small sample size are often met in the process of making disease prediction and could not be solved very well. Support vector machine (SVM), a new kind of machine learning method proposed by Vapnik [19], could solve these problems with many advantages. SVM is an algorithm based on VC dimension theory and structural risk minimization principle. It could solve the small sample, nonlinear problems, high dimension and local minimum points and other practical issues. SVMs are currently being used in many fields, such as text classification [20], [21], image recognition [22], remote sensing information analysis and processing [23], [24], disastrous weather forecast [25], and so on. The prediction models based on SVMs to predict wheat stripe rust timely and accurately could be established.

In the studies on the prediction of wheat stripe rust, many prediction models have been established. However, each model generally has some certain application conditions. Therefore, suitable model should be selected for application according to the actual conditions. Prediction model system for wheat stripe rust could be built to collect the prediction models into one computer system through programming the models. It could make easier to choose suitable model. Integrated prediction model could be established using different models and methods according to the needs in the future.

Acknowledgments. This work was supported in part by Special Fund for Agroscientific Research in the Public Interest (200903035).

\section{References}

1. Li, Z.Q., Zeng, S.M.: Wheat Rusts in China. China Agriculture Press, Beijing (2002) (in Chinese)

2. Chen, X.M.: Epidemiology and Control of Stripe Rust [Puccinia striiformis f. sp. tritici] on Wheat. Can. J. Plant Pathol. 27, 314-337 (2005)

3. Line, R.F.: Stripe Rust of Wheat and Barley in North America: a Retrospective Historical Review. Annu. Rev. Phytopathol. 40, 75-118 (2002)

4. Zeng, S.M.: Interregional Spread of Wheat Yellow Rust in China. Acta Phytopathologica Sinica 18, 119-223 (1988) (in Chinese)

5. Yang, Z.W., Shang, H.S., Pei, H.Z., Xie, Y.L.: Dinamic Forecasting of Stripe Rust of Winter Wheat. Scientia Agricultura Sinaca 24, 45-50 (1991) (in Chinese)

6. Hu, X.P., Yang, Z.W., Li, Z.Q., Deng, Z.Y., Ke, C.H.: Studies on the Prediction of Wheat Stripe Rust Epidemics in Hanzhong District of Shaanxi Province. Acta Univ. Agric. Boreali-occidentalis 28, 18-21 (2000) (in Chinese)

7. Fan, S.Q., Xie, X.S., Li, F., Yin, Q.Y., Zheng, W.Y.: Forecast Model for Prevalent Stripe Rust in Winter Wheat in Shanxi Province. Chinese Journal of Eco-Agriculture 15, 113-115 (2007) (in Chinese) 
8. Chen, G., Wang, H.G., Ma, Z.H.: Forecasting Wheat Stripe Rust by Discrimination Analysis. Plant Protection 32, 24-27 (2006) (in Chinese)

9. Chen, G., Wang, H.G., Zhang, L.D., Wang, T., Ma, Z.H.: Preliminary Research on the Regional Relationship of Epidemic of Puccinia striiformis in China. Chinese Agricultural Science Bulletin 22, 415-420 (2006) (in Chinese)

10. Yun, X.W., Wang, H.G., Ma, Z.H.: Forecast of Wheat Stripe Rust by Upper-air Wind. Chinese Agricultural Science Bulletin 23(8), 358-363 (2007) (in Chinese)

11. Qiang, Z.F.: Markov Forecast of Wheat Stripe Rust in Qinghai in 1998. Plant Protection 25, 19-22 (1999) (in Chinese)

12. Yuan, L., Li, S.Q.: Application of Principal Component Analysis of Wheat Stripe Rust. Computer Engineering and Design 31, 459-461 (2010) (in Chinese)

13. Pu, C.J.: On Periodic Epidemic Regular Pattern and Prediction of Wheat Stripe Rust in Gansu Province. Acta Phytopathologica Sinica 28, 299-302 (1998) (in Chinese)

14. Liu, R.Y., Ma, Z.H.: The Prediction Methodology of Wheat Stripe Rust Using Combination Model Based on GM(1,1). Journal of Biomathematics 22, 343-347 (2007) (in Chinese)

15. Hu, X.P., Yang, Z.W., Li, Z.Q., Deng, Z.Y., Ke, C.H.: Prediction of Wheat Stripe Rust in Hanzhong Area by BP Neural Network. Acta Agriculturae Boreali-occidentalis Sinica 9, 28-31 (2000) (in Chinese)

16. Zhang, J.: Research on Chaotic Characteristics of the Disaster Rate of Crops and Its GABPNN Forecasting Model. Jour. of Northwest Sci-Tech Univ. of Agri. and For(Nat. Sci. Ed.) 34, 63-66 (2006) (in Chinese)

17. Jin, N., Huang, W.J., Jing, Y.S., Wang, D.C., Luo, J.H.: Long-term Meteorological Prediction of Country Wide Wheat Stripe Rust by Genetic Neural Network. Chinese Journal of Agrometeorology 30, 243-247 (2009) (in Chinese)

18. Ma, Z.H.: Plant Disease Epidemiology. Science Press, Beijing (2010) (in Chinese)

19. Vapnik, V.N.: The Nature of Statistical Learning Theory. Springer, New York (1995)

20. Bao, J., Ji, M., Feng, J.: Text Categorization Method Based on Fuzzy Support Vector Machine. Journal of Liaoning Technical University (Natural Science) 29, 974-977 (2010) (in Chinese)

21. Liu, X.L., Ding, S.F., Zhu, H., Zhang, L.W.: Appropriateness in Applying SVMs to Text Classification. Computer Engineering \& Science 32, 106-108 (2010) (in Chinese)

22. Liu, S.: Image Recognition Based on SVM Information Fusion and DSP Parallel Realization. Computer Engineering and Applications 45, 168-170 (2009) (in Chinese)

23. Wang, H.G., Ma, Z.H., Wang, T., Cai, C.J., An, H., Zhang, L.D.: Application of Hyperspectral Data to the Classification and Identification of Severity of Wheat Stripe Rust. Spectroscopy and Spectral Analysis 27, 1811-1814 (2007) (in Chinese)

24. Fu, J.E., Su, Q.X., Pan, S.B., Lu, J.X.: Support Vector Machine Based Groundwater Level Monitoring Model by Using Remote Sensing Images. Journal of Geo-information Science 12, 466-472 (2010) (in Chinese)

25. Wang, D.C., Wang, C.X., Zhu, T.Y., Qin, J.: Support Vector Machines Based Algorithm for the Disastrous Weather Forecast. Journal of Wuhan University of Technology 32, 121124 (2010) (in Chinese) 